\title{
Mechanism of Contrast Formation in Atomic Force Microscopy in Water
}

\author{
Matthew Watkins and Alexander L. Shluger* \\ Department of Physics and Astronomy and London Centre for Nanotechnology, University College London, \\ Gower Street, London, WClE 6BT, United Kingdom \\ WPI-AIMR, Tohoku University, 2-1-1 Kitahira, Aoba, Sendai, 980-8577, Japan
}

(Received 14 July 2010; published 2 November 2010)

\begin{abstract}
We use computer modeling to investigate the mechanism of atomic-scale corrugation in frequencymodulation atomic force microscopy imaging of inorganic surfaces in solution. Molecular dynamics simulations demonstrate that the forces acting on a microscope tip result from the direct interaction between a tip and a surface, and forces entirely due to the water structure around both tip and surface. The observed force depends on a tip structure and is a balance between largely repulsive potential energy changes as the tip approaches and the entropic gain when water is sterically prevented from occupying sites near the tip and surface.
\end{abstract}

DOI: 10.1103/PhysRevLett.105.196101

PACS numbers: 68.08.De, 61.20.Ja, 68.37.Ps

Spectacular recent progress in force spectroscopy and true-atomic resolution imaging using atomic force microscopy (AFM) in solution [1-6] opens the way for even wider applications of the AFM in biology, tribology, colloid systems, nucleation and growth in inorganic and biomimetics processes. It is driven by rapid advances in frequency modulation AFM (FM-AFM) [7] and in our understanding of hydrophobic and hydrophilic interactions $[8,9]$ and friction [10] in solution. In the models proposed for describing these interactions, collective properties of the model systems are averaged over contact areas typically exceeding several square nanometers [9]. The thermodynamics of interfacially confined water near extended molecularly rough surfaces has only been considered recently [11]. Ongoing experimental work continues to stimulate this exciting area of research. Recent work from Khan et al. [12] uses blunt AFM tips to examine the slow dynamical behavior of nanoconfined water and provides a bridge between macroscopic data measured by surface force apparatus and the single atom data probed by NC-AFM. Their data demonstrate the need to carefully consider the materials making contact and the length and time scales probed if important phenomena are not to be overlooked. Molecular simulation approaches, particularly nonequilibrium molecular dynamics techniques, and additional power of current high performance computing platforms, should aid in the interpretation of these data.

For individual sharp tips used for atomically resolved AFM imaging, however, a quite different balance of interactions between tip, surface and solvent may occur. In particular, it is still unclear whether the instrument can directly measure surface topology or only the structure of water near the surface and how the solvation structure of the tip and surface influence the force measurements? Therefore understanding and interpretation of rapidly emerging atomically resolved FM-AFM images requires further development of theoretical models.
To achieve a qualitative understanding of the forces involved in FM-AFM imaging we considered two fully solvated crystalline surfaces-fluorite $\mathrm{CaF}_{2}$ (111) and cubic $\mathrm{MgO}$ (001) having different symmetry and hydrophilicity - and carried out simulations of their interaction with four different nanoclusters modelling AFM tips in aqueous solution. $\mathrm{CaF}_{2}$ is probably the best understood system in UHV [13], and is rather insoluble making it an attractive target to transfer our understanding from the vacuum to the solvated system. $\mathrm{MgO}$ is a particularly good model system with a simple geometry. The systems considered include a cluster model of an AFM tip apex (or nanotip), a surface, and a solvent (water), see Fig. 1. We assumed that the tip apex is likely to be formed from the same material as the surface through accumulation of material during scanning [13] and used a set of representative model nanotips with different polarities of the tip apex and "sharpness" of the tip directed to the surface. An electrolyte atmosphere, which is present experimentally to screen any excess charge in the system, is neglected in our calculation because the neutral surfaces used here lack a net surface charge and the ion concentration near the surface will be very small.

We calculate the free energy change during the approach of the tip to surface over a particular surface location. The force on the tip, which would be transferred to the cantilever in a real AFM setup, is found from the derivative of the free energy change with respect to tip position. We assume that the tip approach is slow and the tip-watersurface system remains in equilibrium at all times; i.e., all motions in the system are faster than the characteristic frequency of tip oscillation (typically $10-100 \mathrm{kHz}$ ), which is well satisfied for our system. Because of the large system size and extensive sampling required, we use a classical force-field approach. The semiquantitative agreement of AFM modeling in UHV demonstrates that the use of simple rigid ion empirical potentials is a good approximation 
for strongly ionic materials [13]. The force fields describing the surfaces and water-surface interactions were taken from published work, but with polarization terms discarded $[14,15]$. To describe water, the TIP4P/2005 model was used [16]. The derivation of the water-surface interactions has been detailed in the literature [14,15]. The setup is similar in spirit to grand canonical molecular dynamics used in [17].

The change in free energy as a tip approaches a surface is calculated using the perturbation method of Zwanzig [18] by performing simulations with a numerical setup similar to those used in Refs. [8,19] using classical molecular dynamics as implemented in GROMACS [20]. Visualization and manipulations of tip coordinates were carried out using the VMD program [21]. Additional computational details are given in the supporting information [22].

A stable $\mathrm{CaF}_{2}$ nanotip model was constructed using $\mathrm{MD}$ anneal and exposes only surface fluoride ions. We considered two different tip orientations of this nanotip (see Fig. 1). The first orientation has a single fluoride ion closest to the surface and will be referred to as the sharper tip; the second, blunter, orientation has two fluoride ions at similar heights. The $\mathrm{CaF}_{2}$ substrate consisted of $192 \mathrm{FCaF}$ units cut out from the bulk fluorite structure to expose two (111) faces and was three FCaF units deep. Each trilayer contained $8 \times 8 \mathrm{CaF}_{2}$ units arranged in a hexagonal lattice. An initial $5 \mathrm{~nm}$ vacuum gap between the two faces of the $\mathrm{CaF}_{2}$ slab was filled with ca. 1500 water molecules. The equilibriated water above the $\mathrm{CaF}_{2}$ (111) surface is layered in the

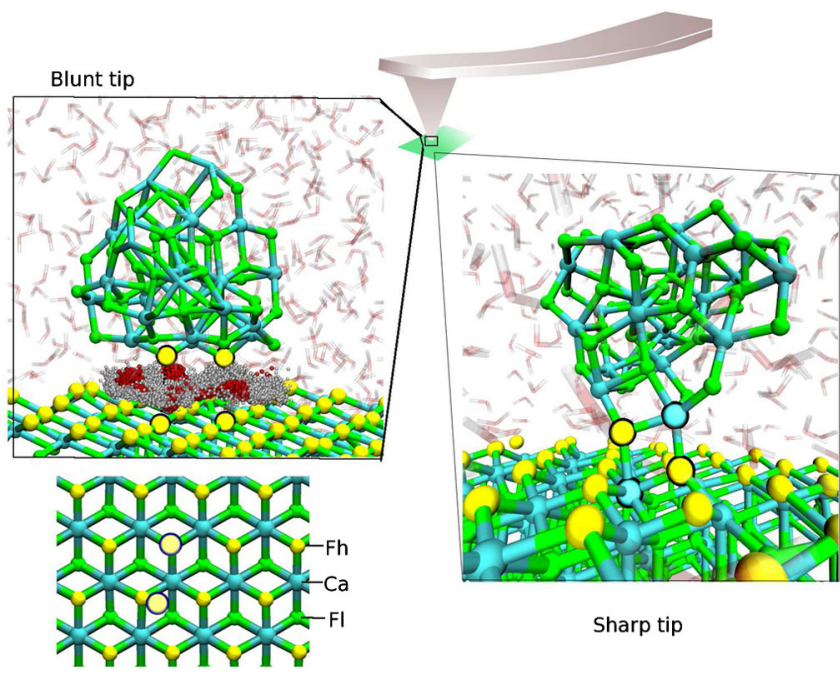

FIG. 1 (color online). Illustrations of the systems considered in this Letter: the structure of the $\mathrm{CaF}_{2}$ substrate and $\mathrm{CaF}_{2}$ nanoparticle. $\mathrm{Ca}$ ions are turquoise, fluoride ions green, except top fluoride (Fh) and tip apex ions which are yellow. The most important tip apex ions and surface ions are highlighted for the blunter tip (left) or the sharper tip (right). Water molecules important in screening the direct-tip-surface interaction are indicated for the blunt tip. A view from above the surface is shown bottom left indicating the surface ion types - the lateral positions of the tip apex ions of the blunt tip are also shown here. direction perpendicular to the surface and within the layers the water density reflects the surface symmetry. Overall, the water structure is perturbed to a distance of around $1 \mathrm{~nm}$ from the surface. Three water layers that exhibit the hexagonal structure of the surface can be clearly identified; moving away from the surface the layer density maxima are above surface $\mathrm{Ca}, \mathrm{Fh}$, and then $\mathrm{Fl}$ ions. The water density above different surface sites is shown in Figs. 2(a) and 3(a). Detailed analysis of the water structure will be published elsewhere [23].

Figs. 2 and 3 show approach curves for the "sharp" and "blunt" tips over calcium (Ca) surface ions and topmost fluorine (Fh), respectively. To better understand the data we have decomposed the interaction into potential energy and entropic contributions [24] [Fig. 2(b) and 3(b)], and "direct" and water mediated components [Figs. 2(c) and 3(c)]. The decomposition into direct and water mediated components is carried out as detailed in the appendix of [8].

In all cases there is an almost entirely attractive entropic contribution to the free energy change. This arises from the increase in translational and rotational degrees of freedom when water initially confined near the tip or surface is forced away into bulklike positions by tip-surface proximity. By contrast, in vacuum the evolution of potential energy is almost indistinguishable from the overall free energy change. The observed force is thus a balance between largely repulsive potential energy changes as the tip approaches and the entropic gain when water is sterically prevented from occupying sites near the tip and surface. The entropic terms increase sharply when the tip encounters higher water densities near the surface.

We find that direct-tip-surface interactions are similar to those found in UHV. For example, for the sharp tip with the fluorine tip apex ion above the $\mathrm{Ca}$ ion of the surface the direct interaction is significant at heights of ca. $0.2-0.4 \mathrm{~nm}$. The atomic configuration at the minima of the direct


FIG. 2 (color online). Approach curves for a sharp $\mathrm{CaF}_{2}$ nanotip to the $\mathrm{CaF}_{2}$ (111) surface over a $\mathrm{Ca}$ ion. (a) water density above a surface $\mathrm{Ca}$ ion without the tip present (b) the free energy change for tip approach decomposed into entropic and potential contributions (c) a decomposition into direct and water mediated contributions (d) the force as the tip approaches the surface over $\mathrm{Ca}$ and also over a surface Fh ion. 
interaction is shown in Fig. 1(a). Conversely, the blunt tip has only a weak direct interaction with the surface, which results from the interactions of several tip and surface ions (the lateral locations of the two main tip terminating fluorine ions are shown in Fig. 1). The overall interaction becomes strongly repulsive before the direct interaction could be measurable: the overall force is totally dominated by the water mediated interaction, Fig. 3(c). The ratio of direct to water mediated force is enhanced for the sharper tip as it displaces less water at a given tip height.

The water mediated interactions are more complex and represent the main difference from UHV. We can roughly divide these interactions in two types. An energetic cost to displace water that is bound to the hydrophilic surface causes variations in the tip-surface force that approximately follow changes in the water density above different surface sites. Figure 2(a) shows that over the surface $\mathrm{Ca}$ ion sites there is relatively little water until a strong peak at ca. $0.2 \mathrm{~nm}$, close to an ideal Ca-F ionic bond length. Over the Fh site the onset of the repulsive interaction occurs at greater tip-surface distance when the third water layer over the $\mathrm{CaF}_{2}$ surface is encountered, Fig. 3(a).

A second type of water mediated interaction occurs from the creation of favorable water locations between tip and surface when they are separated by distances comparable to diameter of a water molecule. In Fig. 3(e) we show the positions of water molecules that have a hydrogen atom within $0.3 \mathrm{~nm}$ of the two tip apex ions of the blunt tip when the tip is at a height of $0.37 \mathrm{~nm}$ above the surface. Figure 1(b) shows another view of this system and also highlights two surface fluorine ions that are under the tip apex ions. The hydrogen ions of the water molecules are



FIG. 3 (color online). (a-c) as Fig. 2, but for the approach of a blunt $\mathrm{CaF}_{2}$ tip over a surface Fh ion. (d) the force curves over surface $\mathrm{Ca}$ and $\mathrm{Fh}$ ions (e) an atomic representation showing the positions of water molecules between the tip and surface (oxygen red, hydrogen silver) when the tip is $0.37 \mathrm{~nm}$ above the surface. Tip atoms other than two apex ions are omitted. Every 2 ps of simulation all water molecules having a hydrogen atom within $0.3 \mathrm{~nm}$ of the highlighted surface fluorine atoms were drawn. Waters orientate to screen the tip and surface fluorine ions. generally located to interact favorably with both the tip and surface fluoride ions, appearing as a sort of halo [Fig. 3(e)], which results in an increase of potential energy. These favorable collective water interactions between tip and surface appear to be a general phenomenon. More detailed atomic configurations, and qualitatively similar configurations above the $\mathrm{MgO}(001)$ surface, are shown in supporting material Figs. S1-3.

The total force curves for the two tips approaching the surface over surface Ca or Fh sites [Figs. 2(d) and 3(d)] demonstrate variations in free energy and force between surface sites of similar magnitude to those observed in vacuum and shown to provide good atomic contrast [13].

To test how general these findings are we carried out a similar study for the $\mathrm{MgO}$ (001) surface. To compare with previous studies in UHV we used a 64 ion cube tip [13] orientated with either an $\mathrm{Mg}$ or an $\mathrm{O}$ ion corner pointing towards the surface. $\mathrm{MgO}$ tips differ from $\mathrm{CaF}_{2}$ in two ways: cationic tip terminations can easily be produced and the tip ions carry different charges. Both tip types are atomically sharp and without large electric multipoles, which has been explored in numerous previous studies [13]. The $\mathrm{MgO}$ (001) surface was constructed from a $7 \times$ $7 \times 3$ bulk supercell and an initial vacuum gap was filled with ca. 2500 water molecules. Figures 4(a) and 4(b) shows the force curves for the $\mathrm{MgO}$ tip approaching the $\mathrm{MgO}$ (001) surface. $\mathrm{MgO}$ is found to be more hydrophilic than $\mathrm{CaF}_{2}$, observed as higher water density near the surface. Generally, the solvent mediated peaks are found to be stronger than for $\mathrm{CaF}_{2}$. Decompositions into "direct and water mediated components [Figs. 4(c) and 4(d)] were carried out as before.

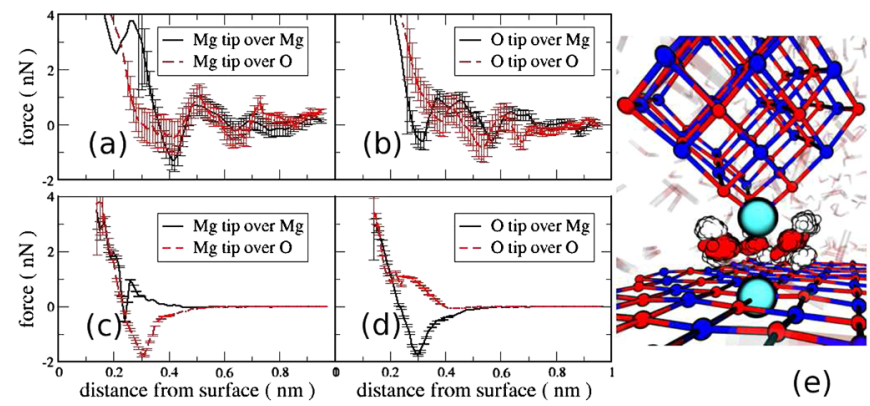

FIG. 4 (color online). Tip force and free energies for the approach of a $\mathrm{MgO}$ cube to a $\mathrm{MgO}$ (001) surface. (a-b) The tip apex atom is centered over one of two surface sites, $\mathrm{O}$ or $\mathrm{Mg}$ and the tip termination is either $\mathrm{Mg}$ or $\mathrm{O}$. Nearer than about $0.26 \mathrm{~nm}$ the tip and surface make chemical or mechanical contact and the perturbation approach taken here is no longer reliable-error bars are very large and omitted for clarity. (c-d) Direct interaction force without solvent interaction between tip and surface for the two tips over surface cation or anion sites. The atomistic model shows the $\mathrm{Mg}$ tip over an $\mathrm{Mg}$ site and the three water molecules that interact particularly favorably with tip and surface (water molecules are drawn in every $20 \mathrm{ps}$ ). $\mathrm{Mg}$ is blue, $\mathrm{O}$ red and $\mathrm{H}$ white/grey. 
The decomposition shows that the direct interactions of the $\mathrm{O}$ tip over an $\mathrm{O}$ site and the $\mathrm{Mg}$ tip over an $\mathrm{Mg}$ site are still strongly repulsive and similar to previously observed in vacuum. However, the total force in solution exhibits a more complex behavior-for an $\mathrm{O}$ tip approaching the surface over an $\mathrm{O}$ site an oscillatory force is observed [Fig. 4(b)] and at relatively large distances from the surface, $0.5 \mathrm{~nm}$, there is a net attractive tip-surface interaction. In the case of the $\mathrm{Mg}$ terminated tip over an $\mathrm{Mg}$ surface ion there is a well defined free energy minimum for the tip at a distance of $0.37 \mathrm{~nm}$ from the surface leading to an attractive tip-surface force at $0.41 \mathrm{~nm}$ [Fig. 4(a)]. At this tip height water molecules can interact with both tip and surface in a favorable manner: attractive interactions occur between water $\mathrm{O}$ atoms and tip and surface $\mathrm{Mg}$ ions and for each water molecule one hydrogen atom is directed towards a tip $\mathrm{O}$ ion and the other to a surface $\mathrm{O}$ ion, Fig. 4(e) shows these water molecules. More detailed views of the atomic configurations at free energy minima between $\mathrm{Mg}$ tip and $\mathrm{Mg}$ surface ion are shown in Fig. S2 and for O tip over O surface $\mathrm{O}$ ions in Fig. S3 of the supplementary material [22]. Another qualitative difference is that in vacuum the direct-tip-surface interactions are very similar for either the $\mathrm{Mg}$ tip approaching $\mathrm{O}$ surface ions, or $\mathrm{O}$ tip nearing $\mathrm{Mg}$ ions, however, the full curves in solution show that this symmetry is broken by water mediated interactions.

Significant effects due to the tip structure and polarity are clearly seen in Figs. 4(a) and 4(b) for different tips approaching over the same surface site. Thus, the measured force is not necessarily a direct measure of the surface and its undisturbed solvation structure. The force curves for the oxygen terminated tip have different distance dependencies over $\mathrm{Mg}$ or $\mathrm{O}$ surface ions and should give rise to contrast in experimental images at a wide range of tip heights. The difference in height dependence of the force curves for the $\mathrm{Mg}$ terminated tip over different surface sites [Fig. 4(a)] is less pronounced, but where the direct force is significant, should still allow atomic resolution.

Our simulations demonstrate that force variations with the tip height and lateral postion arise from a complex convolution of the direct interaction between tip and surface, and forces entirely due to the water structure around both tip and surface. As in vacuum, a sharp tip is needed to sample direct-tip-surface interactions, however, in solution lateral contrast due to the adsorbed water structuring should be measurable even with blunter tips. Decompositions of the free energy changes show a strong entropic contribution, raising the intriguing possibility of tuning the solvent meditated interaction by varying the experimental temperature. Although water is in a dynamic equilibrium at typical tip approach and retraction frequencies of $\mathrm{kHz}$, for even more hydrophilic surfaces, or for more viscous solvents this is likely not the case $[25,26]$. Finally, we note that our results can be extended to other situations where individaul nanoparticles interact with surfaces, such as often occur in microfluidics and the early stages of self-assembly.

M. W. is supported by the Leverhulme Trust (grant F/07 134/CK). The authors are grateful to T. Fukuma, S. Perkin and B. Hoogenboom for stimulating discussions and to A. Foster and A. Kuehnle for valuable comments. Max Berkowitz provided essential guidance into how to address the liquid environment. M.W. would like to thank Changsun Eun for help with PMF calculations.

*matthew.watkins@ucl.ac.uk

[1] T. Fukuma et al., Appl. Phys. Lett. 86, 034103 (2005).

[2] B. W. Hoogenboom et al., Appl. Phys. Lett. 88, 193109 (2006).

[3] M. J. Higgins et al., Biophys. J. 91, 2532 (2006).

[4] T. Fukuma M. J. Higgins, and S. P. Jarvis, Phys. Rev. Lett. 98, 106101 (2007).

[5] S. Rode et al., Langmuir 25, 2850 (2009).

[6] T. Fukuma et al., Phys. Rev. Lett. 104, 016101 (2010).

[7] T. Fukuma and S. P. Jarvis, Rev. Sci. Instrum. 77, 043701 (2006).

[8] C. Eun and M. L. Berkowitz, J. Phys. Chem. B 113, 13222 (2009).

[9] T. Li et al., Phys. Rev. B 75, 115415 (2007).

[10] B. Bhushan, J. N. Israelachvili, and U. Landman, Nature (London) 374, 607 (1995).

[11] J. Mittal and G. Hummer, Faraday Discuss. 146, 341 (2010).

[12] S. H. Khan, G. Matei, S. Patil, and P. M. Hoffmann, Phys. Rev. Lett. 105, 106101 (2010).

[13] W. A. Hofer, A. S. Foster, and A. L. Shluger, Rev. Mod. Phys. 75, 1287 (2003).

[14] N. H. De Leeuw and T. G. Cooper, J. Mater. Chem. 13, 93 (2003).

[15] N. H. de Leeuw and S. C. Parker, Phys. Rev. B 58, 13901 (1998).

[16] J. L. F. Abascal and C. Vega, J. Chem. Phys. 123, 234505 (2005).

[17] J. Gao, W. D. Luedtke, and U. Landman, J. Chem. Phys. 106, 4309 (1997)

[18] R. W. Zwanzig, J. Chem. Phys. 22, 1420 (1954).

[19] N. Choudhury and B. M. Pettitt, J. Am. Chem. Soc. 127, 3556 (2005).

[20] B. Hess et al., J. Chem. Theory Comput. 4, 435 (2008).

[21] W. Humphrey, A. Dalke, and K. Schulten, J. Mol. Graphics 14, 33 (1996).

[22] See supplementary material at http://link.aps.org/ supplemental/10.1103/PhysRevLett.105.196101.

[23] M. Watkins, M. L. Berkowitz, and A. L. Shluger (to be published).

[24] The decomposition into internal energy and entropic terms is simply achieved by subtracting the potential energy change of the system from the free energy change.

[25] W. Hofbauer et al., Phys. Rev. B 80, 134104 (2009).

[26] L. T. W. Lim, A. T. S. Wee, and S. J. O'Shea, J. Chem. Phys. 130, 134703 (2009). 